\title{
CAMBIOS DE RANGO TAXONÓMICO Y NUEVAS COMBINACIONES NOMENCLATURALES EN TRES COMPUESTAS MEXICANAS
}

\author{
José Á. Villarreal-Quintanilla ${ }^{1}$ y Andrés E. Estrada-Castillón² \\ ${ }^{1}$ Universidad Autónoma Agraria Antonio Narro, Departamento de Botánica, \\ 25315 Buenavista, Saltillo, Coahuila, México. \\ javillarrea100@hotmail.com \\ ${ }^{2}$ Universidad Autónoma de Nuevo León, Facultad de Ciencias Forestales, \\ Apdo. postal 41, 67700 Linares, Nuevo León, México. \\ aeduardoestradac@prodigy.net.mx
}

\section{RESUMEN}

Se proponen tres nuevas combinaciones nomenclaturales en compuestas mexicanas, consideradas como necesarias para ajustar el rango taxonómico de: Acourtia dugesii (A. Gray) Reveal \& R. M. King var. veracruzana (B. L. Turner) Villarreal \& A. E. Estrada, Acourtia dugesii (A. Gray) Reveal \& R. M. King var. queretarana (B. L. Turner) Villarreal \& A. E. Estrada y Chaptalia lyratifolia Burkart var. estribensis (G. L. Nesom) Villarreal \& A. E. Estrada.

Palabras clave: Acourtia, Chaptalia, Compositae, México.

\section{ABSTRACT}

Acourtia dugesii (A. Gray) Reveal \& R. M. King var. veracruzana (B. L. Turner) Villarreal \& A. E. Estrada, Acourtia dugesii (A. Gray) Reveal \& R. M. King var. queretarana (B. L. Turner) Villarreal \& A. E. Estrada and Chaptalia lyratifolia Burkart var. estribensis (G. L. Nesom) Villarreal \& A. E. Estrada, are proposed as taxonomic rank changes and new nomenclatural combinations for three Mexican taxa of Compositae.

Key words: Acourtia, Chaptalia, Compositae, Mexico. 
Como parte de la preparación de la tribu Mutisieae (sensu lato) de la familia Compositae (Asteraceae) para la Flora de Veracruz, surgió la necesidad de proponer cambios en el rango taxonómico y en la nomenclatura de algunas especies, los cuales se presentan a continuación.

Perezia dugesii A. Gray, fue descrita como nueva en 1883 con base en materiales procedentes del oeste de Guanajuato (Bacigalupi, 1931) y reubicada en el género Acourtia por J. L. Reveal y R. M. King en 1973. La especie tiene una distribución amplia en el centro de México que incluye los estados de Guerrero, Guanajuato, Hidalgo, Jalisco, México, Michoacán, Oaxaca, Puebla, Querétaro y Veracruz (McVaugh, 1984; Turner, 1993). Se caracteriza por sus cabezuelas agrupadas en glomérulos, flores con el labio superior tridentado y el inferior bidentado y las hojas amplexicaules, con aurículas redondeadas. Las variantes morfológicas que conforman este taxon han sido distinguidas como variedades desde el trabajo de Bacigalupi (1931) y más recientemente una serie de especies nuevas del mismo conjunto fue propuesta por Turner (1993). En nuestro criterio, Acourtia dugesii está formada por cuatro variedades: la típica, con cabezuelas sésiles a casi sésiles, con 5-10 flores, que se localiza en la región oeste de su área de distribución; la variedad pilulosa Bacig., con aquenios densamente pubescentes, con hojas lobadas o unilobadas que crece en los estados de Oaxaca y Puebla; y las dos variedades aquí propuestas.

Por otro lado, Chaptalia lyratifolia fue descrita por A. Burkart en 1944, de la parte septentrional de la Sierra Madre Oriental. Las hojas liradas, gruesas, con el lóbulo terminal agrandado, escapos florales ebracteados y la presencia de rizomas en la planta son características distintivas de la especie. Se distribuye en Coahuila, Nuevo León, San Luis Potosí, Tamaulipas y al parecer también en Hidalgo, Oaxaca y Veracruz.

\section{COMBINACIONES PROPUESTAS}

Acourtia dugesii (A. Gray) Reveal \& R. M. King var. veracruzana (B. L.Turner) Villarreal \& A. E. Estrada, comb. nov. Acourtia veracruzana B. L. Turner, Phytologia 74: 408. 1993. Tipo: México, Veracruz, Maltrata, E. Matuda 1236 (Holotipo: MEXU; isotipos: LL, MICH).

La variedad propuesta está basada en Turner (1993), quien diferencia de Acourtia dugesii var. dugesii a las plantas de Veracruz y de áreas aledañas de Hi- 
dalgo y Puebla, por sus cabezuelas con pedúnculos de 2-15 $\mathrm{mm}$ de largo y por sus brácteas del involucro angostamente acuminadas. La revisión de materiales de los herbarios ENCB, MEXU y XAL mostró que un gran número de ejemplares del este de México presenta estas características, sin embargo en la misma comarca existen otros con cabezuelas sésiles o sésiles y pedunculadas en el mismo individuo, y brácteas involucrales acuminadas. Tal variación es común en las plantas que crecen en la región mencionada, por lo que proponemos la categoría de variedad para las poblaciones en cuestión.

Acourtia dugesii (A. Gray) Reveal \& R. M. King var. queretarana (B. L.Turner) Villarreal \& A. E. Estrada, comb. nov. Acourtia queretarana B. L. Turner, Phytologia 74: 402. 1993. Tipo: México, Querétaro, Mpio. Pinal de Amoles, $13 \mathrm{~km}$ al NE de Pinal de Amoles, sobre la carretera a Jalpan, J. Rzedowski 48108 (Holotipo: TEX).

Se trata de poblaciones de $A$. dugesii con distribución en el noreste de Guanajuato y norte de Querétaro, diferenciada por Turner (1993) de la variedad típica por llevar cabezuelas con mayor número de flores (18-20 vs. 5-6), con involucros estrechamente campanulados (vs. cilíndricos) y pedunculadas (vs. sésiles a casi sésiles). Algunos ejemplares provenientes del área mencionada presentan tales características, mientras que otros de la misma región muestran rasgos intermedios. Dada tal variación se propone reconocer el nivel de variedad para este grupo de plantas.

Chaptalia lyratifolia Burkart var. estribensis (G. L. Nesom) Villarreal \& A. E. Estrada, comb. nov. Chaptalia estribensis G. L. Nesom, Phytologia 78: 160. 1995. Tipo: Mexico, Hidalgo, Mpio. Tenango de Doria, 8-11 km al SW de Tenango de Doria, D. E. Breedlove 59571 (Holotipo: CAS, isotipos: ENCB, MEXU, MO).

La variedad propuesta se define mediante una población en el norte de Veracruz, adyacente a Hidalgo y otra registrada en el sur de Oaxaca. Se reconoce por su tendencia de presentar hojas con un lóbulo terminal solitario, con extensiones liradas reducidas o ausentes hacia el pecíolo y pubescencia de color amarillento en el envés. La delimitación original de C. estribensis marca hojas sin extensiones liradas y pubescencia café-anaranjada en el envés, sin embargo, algunas muestras presentan extensiones liradas cortas y pubescencia amarillenta que podría concordar más con lo descrito para la variedad típica de C. lyratifolia. 


\section{LITERATURA CITADA}

Bacigalupi, R. 1931. A monograph of the genus Perezia section Acourtia. Contr. Gray Herb. 97: $1-78$.

Burkart, A. 1944. Estudio del género de compuestas Chaptalia con especial referencia a las especies argentinas. Darwiniana 6: 505-594.

McVaugh, R. 1984. Perezia. Flora Novo-Galiciana 12: 683-702.

Nesom, G. L. 1995. Revision of Chaptalia (Asteraceae: Mutisieae) from North America and continental Central America. Phytologia 78: 153-188.

Reveal, J. L. y R. M. King. 1973. Re-establishment of Acourtia D. Don (Asteraceae). Phytologia 27: 228-232.

Turner, B. L. 1993. New taxa, new combinations, and nomenclatural comments on the genus Acourtia (Asteraceae, Mutisieae). Phytologia 74: 385-413.

Recibido en abril de 2009.

Aceptado en diciembre de 2009. 